\title{
Extracorporeal membrane oxygenation (ECMO) in patients with severe COVID-19 adult respiratory distress syndrome: a systematic review and meta-analysis
}

\author{
Hany Elsayed ${ }^{1}$, Aly Hassaballa ${ }^{1}$, Taha Ahmed ${ }^{1}$, Mohammed Gumaa $^{1}$, and Hazem \\ Sharkawy ${ }^{1}$ \\ ${ }^{1}$ Ain Shams University Faculty of Medicine
}

September 11, 2020

\begin{abstract}
Background COVID 19 is the most recent cause of Adult respiratory distress syndrome ARDS. Extracorporeal membrane oxygenation (ECMO) can support gas exchange in patients failing conventional mechanical ventilation, but its role is still controversial. We conducted a systematic review and meta-analysis on ECMO for COVID-associated ARDS to study its outcome. Materials and Methods CENTRAL, MEDLINE/PubMed, Cochrane Library, and Scopus were systematically searched from inception to May 28, 2020. Studies reporting five or more patients with COVID 19 infection treated venovenous with ECMO were included. The main outcome assessed was mortality. Baseline, procedural, outcome, and validity data were systematically appraised and pooled with random-effect methods. The validity of all the included observational studies was appraised with the Newcastle Ottawa scale. Meta-regression and publication bias were tested. This trial was registered with PROSPERO under registration number CRD42020183861 Results From 1647 initial citations, 34 full text articles were analysed and 12 studies were selected, including 194 patients with confirmed COVID 19 infection requiring ICU admission and venovenous ECMO treatment. Random-effect pooled estimates suggested an overall in-hospital mortality risk ratio of 0.49 (95\% confidence interval 0.259 to 0.721 ; I $=94 \%$ ). Subgroup analysis according to country of origin showed persistent heterogeneity only in the 7 Chinese studies with pooled estimate mortality risk ratio of $0.66(\mathrm{I} 2=87 \%)(95 \% \mathrm{CI}=0.39-0.93)$, while the later larger studies coming from the USA showed pooled estimate mortality risk ratio of 0.41 (95\% CI 0.28-0.53) with homogeneity ( $\mathrm{p}=0.67$ ) similar to France with a pooled mortality risk ratio of 0.26 (95\% CI 0.08-0.43) with homogeneity $(\mathrm{p}=0.86)$.
\end{abstract}

Extracorporeal membrane oxygenation (ECMO) in patients with severe COVID-19 adult respiratory distress syndrome: a systematic review and meta-analysis

Authors:

1. Hany Hasan Elsayed FRCS Cth: Thoracic surgery department (ARDS taskforce) - Ain Shams University - Cairo - Egypt.

2. Aly Sherif Hassaballa MSc:Cardiothoracic surgery department - Ain Shams University - Cairo Egypt.

3. Taha Aly Ahmed MSc:Cardiothoracic surgery department - Ain Shams University - Cairo - Egypt.

4. Mohamed Gumaa MSc: Medical statistics department - Ain Shams University - Cairo - Egypt.

5. Hazem Youssef Sharkawy MBBcH: Cardiothoracic surgery department - Ain Shams University - Cairo - Egypt.

Keywords: COVID 19, ECMO, ARDSCorresponding author: Hany Hasan Elsayed Thoracic surgery department, Faculty of Medicine, Ain Shams University Abbasia square, Cairo Egypt 002/01227373270drhany.elsayed@yahoo.co.uk 
Word count: 4391

\section{Background}

COVID 19 is the most recent cause of Adult respiratory distress syndrome ARDS. Extracorporeal membrane oxygenation (ECMO) can support gas exchange in patients failing conventional mechanical ventilation, but its role is still controversial. We conducted a systematic review and meta-analysis on ECMO for COVIDassociated ARDS to study its outcome.

\section{Materials and Methods}

CENTRAL, MEDLINE/PubMed, Cochrane Library, and Scopus were systematically searched from inception to May 28, 2020. Studies reporting five or more patients with COVID 19 infection treated venovenous with ECMO were included. The main outcome assessed was mortality. Baseline, procedural, outcome, and validity data were systematically appraised and pooled with random-effect methods. The validity of all the included observational studies was appraised with the Newcastle Ottawa scale. Meta-regression and publication bias were tested. This trial was registered with PROSPERO under registration number CRD42020183861

\section{Results}

From 1647 initial citations, 34 full text articles were analysed and 12 studies were selected, including 194 patients with confirmed COVID 19 infection requiring ICU admission and venovenous ECMO treatment. Median New Castle Ottawa scale was 6 indicating acceptable study validity. 136 patients reached an endpoint of weaning from ECMO or death while the rest were still on ECMO. The median Berlin score for ARDS prior to starting ECMO was III. Patients received mechanical ventilation before ECMO implementation for a median of four days and ECMO was maintained for a median of 13 days. In hospital and short-term mortality were highly variable among the included studies ranging between $0 \%$ and $100 \%$. Random-effect pooled estimates suggested an overall in-hospital mortality risk ratio of 0.49 (95\% confidence interval 0.259 to 0.721 ; I $2=94 \%$ ). Subgroup analysis according to country of origin showed persistent heterogeneity only in the 7 Chinese studies with pooled estimate mortality risk ratio of $0.66(\mathrm{I} 2=87 \%)(95 \% \mathrm{CI}=0.39-0.93)$, while the later larger studies coming from the USA showed pooled estimate mortality risk ratio of $0.41(95 \%$ CI 0.28-0.53) with homogeneity ( $\mathrm{p}=0.67)$ similar to France with a pooled mortality risk ratio of $0.26(95 \%$ CI $0.08-0.43)$ with homogeneity $(\mathrm{p}=0.86)$. Meta-regression showed only younger age as a predictor of mortality $(\mathrm{p}=0.02)$. Publication bias was excluded by visualizing the funnel plot of standard error, Egger's test with $\mathrm{p}=0.566$ and Begg\&Mazumdar test with $\mathrm{p}=0.373$

\section{Conclusion}

The study included the largest number of patients with outcome findings of ECMO in this current pandemic. Our findings showed that the use of venovenous ECMO at high-volume ECMO centres may be beneficial for selected COVID 19 patients with severe ARDS. However, none of the included studies involve prospective randomized analyses; and therefore, all the included studies were of low or moderate quality according to the Newcastle-Ottawa scale. In the current era and environment of the pandemic, it will likely be very challenging to conduct a prospective randomized trial of ECMO versus no-ECMO for COVID-19. Therefore, the information contained in this systematic review of the literature is valuable and provides important guidance.

\section{Introduction}

Coronavirus disease 2019 (COVID-19) is a viral respiratory tract infection caused by a coronavirus which was first documented in Wuhan, China, in December 2019 (1)

After then, this outbreak spread globally and has been considered as a pandemic and an international public health emergency by the WHO since March 11, 2020. As of 6th of September 2020, a cumulative total of 27,083,427 confirmed cases of coronavirus disease 2019 (COVID-19) were reported with total 884,029 deaths in 203 countries and territories worldwide (2). Currently, there is neither a proven effective medication nor a vaccine has been discovered for the COVID-19 infection. 
Although most patients with COVID-19 infection have only mild or uncomplicated course, around 10-20\% will develop a severe disease that necessitates hospitalization and oxygen therapy or even ICU admission and progression to acute respiratory distress syndrome (ARDS). The prevalence of ARDS caused by COVID-19 is around $8.2 \%$ who will require mechanical ventilation and prone positioning (3) . Furthermore, hyperinflammatory state by cytokine storm appears to be a solid part of severe COVID-19 disease (4)

However, a group of patients will suffer from persistent hypoxemia and intractable ARDS despite maximum conventional treatment with mechanical ventilation and mortality among this subgroup is markedly high. Initial reports from China, Italy and USA suggest high patient admission to intensive care units (ICU) and mechanical ventilation with shockingly very high mortality rate among patients with severe ARDS due to COVID 19

\section{$(5-9)$}

Another option for severe refractory ARDS patients is venovenous extracorporeal membrane oxygenation (ECMO) which is considered as a rescue therapy. ECMO appeared to be beneficial during the influenza A (H1N1) pandemic back in 2009, with a mortality rate of $21 \%$ (10). In another observational study on patients with $\mathrm{H} 1 \mathrm{~N} 1$ related ARDS, the mortality rate was $23.7 \%$ for ECMO patients in versus $52.5 \%$ for non ECMO patients (11). In 2018, a retrospective study was conducted on middle east respiratory syndrome (MERS) patients with refractory ARDS and showed that ECMO should be used as a rescue therapy because it is associated with lower mortality when compared to conventional mechanical ventilation group (65 vs. $100 \%, \mathrm{P}=0.02)(12)$. No ECMO report was published on SARS (coronavirus emerging in 2002) related ARDS.

The role of ECMO in the management of COVID- 19 ARDS remains unclear. The initial reports of using venovenous ECMO with COVID- 19 patients suffering from intractable hypoxemia observed a high mortality rate and recommended using ECMO with caution in the current pandemic (13). According to the interim guidance made by the World Health Organization (WHO), venovenous ECMO could be considered as a salvage therapy for COVID-19 with refractory hypoxemia in expert centers with enough cases to ensure clinical expertise (14) .

In view of the current growing pandemic and the fact that only a little experience with using ECMO to support COVID-19 patients is available, we aimed to estimate the effect of venovenous ECMO on mortality from COVID-19 patients with respiratory failure via all available studies by performing a systematic review and meta-analysis.

\section{Methods}

\section{Search strategy and selection criteria}

Our methodology followed the reporting guidelines of Meta-analysis Of Observational Studies in Epidemiology (MOOSE) and Preferred Reporting Items for Systematic Reviews and Meta-Analyses (PRISMA) guidelines. We electronically ran a search on CENTRAL, MEDLINE/PubMed, Cochrane Library, and Scopus. On Pubmed the word search used was (COVID OR SARS COV2 OR pandemic) AND (ARDS) OR (acute respiratory distress syndrome) OR (acute lung injury) OR (respiratory failure) OR (respiratory insufficiency) OR (ECMO) OR (extracorporeal membrane oxygenation)).

We contacted authors for some missing data, searched trial registries, included the grey literature, and used studies accepted and ahead of print. We did our search from inception up to May 28, 2020, which was the date of our final search without language restrictions. We used both subject headings and text word terms to search for articles about ECMO or mechanical ventilation with ARDS in COVID-19 patients. There were no language restrictions. Inclusion criteria were (all criteria should be concomitantly met for study inclusion): a) study reporting on 5 or more patients with final outcomes; b) with confirmed COVID 19 infection; c) receiving venovenous ECMO. Exclusion criteria were (one criterion was sufficient for study exclusion): a) inclusion of $<5$ patients with COVID-19 infection treated ECMO (thereby, any study reporting on fewer than 5 patients or case reports treated with ECMO were excluded); b) duplicate publication (in which case only 
the most recent report from the same study group was included in the systematic review). Use of a sample size cut-off was chosen to limit the risk of imprecision and publication bias $c$ ) Studies in which the main focus was veno-arterial ECMO for treating COVID-19 or $d$ )studies with insufficient data about outcome endpoints (mortality, extubation, weaning, and discharge). AH, TA and HY independently reviewed the titles and abstracts of all citations. Then, they independently reviewed the full text of both definite and potentially eligible studies for inclusion. Disagreements were reviewed by a fourth reviewer HE, who had a deciding vote. The study protocol link is at www.crd.yorl.ac.uk/PROSPERO under registration number CRD42020183861

\section{Quality assessment}

The quality of the included studies was assessed using the Newcastle-Ottawa scale (NOS) (15). This assessment tool is recommended by the Cochrane collaboration to assess risk of bias of non randomised observational studies. The tool uses three domains: selection of study groups (four points); comparability of groups (two points); and ascertainment of exposure and outcomes (three points). The higher the score the less is the risk of bias. The maximum score on the Newcastle-Ottawa scale is 9 . Thresholds for converting the Newcastle-Ottawa scales to AHRQ standards (good, fair, and poor):

"-Good quality: three or four stars in selection domain + one or two stars in comparability domain + two or three stars in outcome/exposure domain

-Fair quality: two stars in selection domain + one or two stars in comparability domain + two or three stars in outcome/exposure domain.

-Poor quality: zero or one star in selection domain OR zero stars in comparability domain OR zero or 1 stars in outcome/exposure domain."

\section{Data analysis}

A meta-analysis was conducted to examine the mortality incidence in venovenous ECMO treatment for COVID 19. Data were summarized using the risk ratio (95\% confidence interval (CI)). The data were pooled using DerSimonian-Laird random effects model (16). P value of 0.05 or less was statistically significant. Cochran Q and I2 were used to assess heterogeneity between studies. The degree of heterogeneity was categorized as either low (I2 $<25 \%$ ), moderate (I2 $=25 \%-75 \%$ ), or high (I2 $>75 \%$ ) (17). A P value of [?] 0.05 indicated significant heterogeneity. A subgroup meta-analysis according to the study's country of origin was conducted to investigate the high heterogeneity detected. Exploratory meta-regression analysis was performed to identify significant moderators using the inverse-variance weighted-least-squares linear regression analysis. Studies that included a control group with patients treated with mechanical ventilation were independently studied and mortality estimates were pooled using odds ratio and $95 \%$ CI. Secondary outcome measures included age, ventilation days before ECMO, and duration time on ECMO. Publication bias was examined by visual inspection of the funnel plot and tested by Egger's test and Begg and Mazumdar test. A P value of [?] 0.05 indicated the existence of publication bias. All analyses were performed using Open Meta Analyst software Windows 10 version.

The data used in the meta-analysis in each study were the number of mortality events and the number of closed cases (either cured or dead). Patients who were still on treatment were not included in the final analysis of cases of the study. The corresponding authors of the studies were contacted by email to provide additional information regarding the patients who were still receiving treatment. The numbers used in Jacobs et al (2020) and Beyls et al (2020) were not those reported by the study but rather provided by the author (unpublished data).

Trial was registered with PROSPERO at www.crd.yorl.ac.uk/PROSPERO under registration number CRD42020183861

\section{Role of funding source}


There was no funding source for this study. The corresponding author of this study had full access to all the study data and had final responsibility for the decision to submit the manuscript for publication.

Results

Our electronic search retrieved 1647 citations, 34 of which were selected for full-text review (Figure 1). Twelve studies with a combined population of 194 patients fulfilled the inclusion criteria (Table 1). 136 patients reached an endpoint of weaning from ECMO or death while the rest were still on ECMO. Overall study validity was acceptable, with a median score of 6 on the Newcastle Ottawa scale NOS appraising the quality of observational studies, without being opposed by their non-randomized design. The median Berlin score for ARDS prior to starting ECMO was III. Patients received mechanical ventilation before ECMO implementation for a median of four days and ECMO was maintained for a median of 13 days (Table 2,3)

Random-effect pooled estimates suggested an overall in-hospital mortality risk ratio of 0.49 (95\% confidence interval 0.259 to $0.721 ; \mathrm{I} 2=94 \%$ ) (Figure 2 ). Most of the preliminary studies were from China (seven studies with 41 patients having endpoints). Larger studies then followed from the USA, Japan and France (Five studies with 95 patients with endpoints).

To investigate the overall inter-study heterogenicity, a subgroup analysis was performed according to the country of origin of each study (Figure 3) This showed persistent heterogeneity only in the 7 Chinese studies with pooled mortality risk ratio of $0.66(\mathrm{I} 2=87 \%)(95 \% \mathrm{CI}=0.39-0.93)$, while the later larger studies coming from the USA showed pooled estimate mortality risk ratio of 0.41 (95\% CI 0.28-0.53) with homogeneity $(\mathrm{p}=0.67)$ similar to France with a pooled mortality risk ratio of 0.26 (95\% CI $0.08-0.43)$ with homogeneity $(\mathrm{p}=0.86)$

In four of our studies, there was a control group who received mechanical ventilation for severe ARDS. The mortality rate was $87.5 \%$ in the ECMO patients and $69.2 \%$ in conventional therapy patients. The pooled odds of mortality in ECMO versus conventional therapy were not significantly different $(\mathrm{p}=0.273,95 \% \mathrm{CI}$ : 0.06-1.111). There was no observable heterogeneity ( $\mathrm{I} 2=0 \%$, Cochran's $\mathrm{Q}, \mathrm{p}$-value $=0.57$ ( (Figure 4)

Moderators tested were age, Pre ECMO-Berlin score of ARDS, ventilation days before ECMO, and duration of days on ECMO. Exploratory meta-regression identified age as a significant negative moderator of mortality $(\mathrm{P}=0.02)$ with younger age patients at a higher risk of death. No other factors demonstrated a significant moderator but this lack of a statistical significance for likely or established prognostic factors should be viewed with consideration of the limited statistical power of meta-regression when applied to a limited dataset. Publication bias was excluded by visualizing the funnel plot of standard error (Figure 5). The funnel plot is symmetrical with only 2 studies outside the threshold $(20,23)$. Exclusion of bias was also proven with an Egger's test value $\mathrm{p}=0.566$ and Begg\&Mazumdar test value with $\mathrm{p}=0.373$

\section{Discussion}

To our knowledge, this is the first systematic review and meta-analysis of the use of veno venous ECMO in treating severe COVID 19 related ARDS. We identified 12 independent studies (18-29) that provided data on the outcome of ECMO in COVID 19 patients. The initial studies testing the efficacy of ECMO in COVID 19 were small and pessimistic. A study by Namendys-Silva SA (30) looked at pooled mortality and efficacy from initial Chinese reports (only 17 patients). It included 2 of our included studies $(22,25)$ and found a mortality of $82.3 \%(14 / 17)$ with no overall benefit of ECMO ( $\mathrm{z}=0.57, \mathrm{p}=0.56)$. Henry and Lippi (31) shortly followed by another pooled analysis which included also 2 of our included studies $(22,23)$ comparing ECMO (total of 17 patients) and conventional mechanical ventilation and again found no benefit for ECMO in severe COVID 19 with pooled odds of mortality in the ECMO group versus the conventional therapy group not significantly different (OR: 2.00, 95\%CI: 0.49-8.16) with no observable heterogeneity (I2 $=0 \%$, Cochran's $\mathrm{Q}, \mathrm{p}$-value $=0.99)$.

We performed a subgroup single arm meta-analysis for studies including another arm for invasive mechanical ventilation for severe ARDS due to COVID 19, but all our ECMO studies had 5 or more patients (22-25) 
as per inclusion criteria and again found no benefit or harm for ECMO in treating severe ARDS in COVID 19. The pooled odds of mortality in the ECMO group versus the conventional therapy group were not significantly different $(\mathrm{p}=0.273,95 \% \mathrm{CI}$ : $0.06-1.111)$. The mortality was high in both groups $(87.5 \%$ vs $69.2 \%$ ). We believe there are two limitations here, firstly, none of the larger more recent studies with better outcomes $(20,21,26-29)$ had a control arm of treating COVID related ARDS with conventional mechanical ventilation. Secondly, we believe these are two different populations with patients having variable levels of ARDS severity, with those receiving ECMO treatment being potentially more critically ill in some cases, which could have impacted the outcome for mortality rates.

We have shown by our subgroup analysis that heterogeneity was mainly in the initial Chinese (I2=87\%) studies and the following studies showed homogeneity from the USA (I2 $=0 \%, \mathrm{P}=0.67)$ and France (I2 $=0 \%$, $\mathrm{p}=0.86$ ) with lower mortality associated with the use of ECMO for severe COVID 19 patients, although the number of studies was small. The three largest case series $(20,21,27)$ included in this systematic review were all outside China and showed more promising results than the rest of studies with smaller numbers $(18,19,22-26,28,29)$. A mortality of $29.2 \%$ only in these series as compared to a mortality of $61.1 \%$ in the rest of the nine studies (seven Chinese series). This may reflect the fact that high flow ECMO centres achieve superior results. Our data thus confirm these results showing the beneficial effects of ECMO in large tertiary referral centres for the treatment of COVID 19 ARDS. This is consistent with previous findings of Barbaro and his colleagues (32) who found lower ECMO case-mix adjusted mortality in adult patients (adjusted OR, 0.61; 95\% CI, 0.48-0.79) in higher volume ECMO centres. Our definition of high volume centres is like them as performing more than 30 cases/year.

The role of ECMO in improving outcomes in severely ill COVID 19 patients seems to be multi factorial. The ARDS observed in COVID 19 patients mostly fits the Berlin criteria (33) but Gattinoni and his colleagues (34) have proposed that the classic ARDS injury is only present in 20-30\% of COVID 19 patients with decreased pulmonary compliance less than $40 \mathrm{ml} / \mathrm{cmH} 2 \mathrm{O}$ while the non-ARDS type (present in 70-80\%), the severe hypoxemia is associated with a respiratory system compliance of more than $50 \mathrm{ml} / \mathrm{cmH} 2 \mathrm{O}$. Hence, severe hypoxemia is primarily due to ventilation/perfusion (VA/Q) mismatch. Unlike classic ARDS, high PEEP pressures and prone positioning in this subgroup of COVID-19 patients do not improve oxygenation through the classic theory of the recruitment of collapsed areas. Although our meta regression showed that pre ECMO days of ventilation did not affect outcome, these patients could benefit from early ECMO to avoid ventilator-induced lung injury and this was the recommendation from the only two included studies in our analysis which had zero mortality in their ECMO patients $(18,20)$

Secondly, there is an increasing trend to show that COVID 19 infection is associated with a hypercoagulable and thrombotic state. Yin et al (35) studied the differences of coagulation features between severe pneumonia caused by the SARS-CoV-2 (COVID 19) and non-SARS-CoV-2 viruses and found that platelet count of the COVID19 group was significantly higher than that of non-COVID19 patients. Beyls and his colleagues (27) suggested that venous-Doppler ultrasonography of femoral and jugular veins should be performed routinely for severe COVID-19-related ARDS in preparation in case ECMO therapy is needed as they found a higher rate of ECMO related line thrombosis. ECMO circuits eliminate coagulation factors binding them irreversibly to their surface coating material. Systemic anticoagulation is usually utilized for ECMO and further aggravates the anti-coagulatory state on many levels (36). In their current COVID-19 guidelines, the "Extracorporeal life support organisation ELSO" recommended following existing anti-coagulation guidelines, with consideration given to an anti-coagulation targeted at the higher end of normal with vvECMO given the known hyper-coagulable status of COVID-19 patients (37)

In their meta-analysis Munshi and his colleagues (38) have shown a reduction in 60 day mortality in patients receiving ECMO for ARDS in comparison to conventional mechanical ventilation with an associated increased risk of bleeding. The improvement in ARDS outcome and the anti-thrombotic benefit shown in this metaanalysis is a hypothesis of the additional benefit in severely ill COVID 19 patients.

Two of our studies $(21,23)$ suggested that higher mortality related to patients receiving ECMO or conventional mechanical ventilation for severe ARDS can be related to cytokine production. There is accumulating 
evidence suggesting that a sub-group of patients with severe COVID-19 disease have a cytokine storm syndrome in which a cascade of activated cytokines leads to harmful auto-amplification of inflammatory cytokine production leading to end-organ damage and increasing the risk of mortality. Among COVID-19 patients who have received ECMO, a strong positive correlation exists between mortality and high cytokine levels, most notably IL-6 (39).

Ruan et al (23) found that Interleukin-6 concentrations differed significantly between non survivors and survivors in their COVID-19 cohort, with non survivors having up to 1.7 times higher values. We could not study this in our meta-analysis as both studies did not mention absolute values for cytokines for the ECMO only group.

Our meta-analysis suggests that there is some potential role for ECMO in appropriately selected patients with severe COVID-19. Although the risk factors and variables that contribute to the optimal outcome are complex and reflect individual ECMO center experiences and available resources during the pandemic, it can be argued that it would be unethical to withhold ECMO (or consideration for referral to an experienced ECMO center) in patients who might potentially benefit from this therapy as suggested by Abrams and colleagues (40) when considering ECMO for ARDS due to all causes.

The planning and execution of a randomised controlled trial of this advanced intervention during the current pandemic is difficult. Current challenges include randomisation of markedly sick patients early with a higher risk of death, the need for engagement of many centres worldwide, the lack of the ECMO service in poorer and third world countries and the inconsistency of managing the control non ECMO group. As a result, a current study of ECMO in patients with COVID 19 related severe ARDS soon is unlikely. Thus, our metaanalysis can provide clinicians with the most comprehensive synthesis of all the available limited evidence for the outcome of vvECMO in adult patients with severe ARDS due to COVID-19, although further data collection and meta-analysis for larger studies are invited.

\section{Limitations}

The project has obvious limitations, including those which are typical of any systematic review and metaanalyses. By pooling observational studies, this review cannot overcome the limitations of its primary studies included which were relatively of small numbers ( apart from one (21)) and, still none were based on a randomised allocation. Indeed, the authors believe only meta-analyses of homogeneous well-powered randomised trials should be considered a solid scientific proof of the safety and efficacy of any medical/surgical intervention which is difficult to achieve on the short term period while we are still facing an unresolved pandemic and literature guidance from the available data is needed to support decision on a stretched medical resources setting in most countries The secondary outcome data was missing in numerous studies and the focus by the authors was mortality outcome and hence authors needed to be contacted by emails to fill in missing information. However, systematic reviews and meta-analyses of non-randomized studies (as in the current case) can be meaningful and guide current practice, even if only by emphasizing the limitations of the available clinical evidence (as in the current ECMO use with the COVID 19 pandemic).

Furthermore, the exclusion of 14 reports because including less than 5 cases or being case reports is a call for more collaborative research efforts. This type of collaboration is essential for the present clinical challenges of the COVID 19 crisis. To complement this collaboration, ASAIO has developed a database specific to ECMO use in severe COVID-19 to aid in this effort. Merging and synergizing data between databases such as those obtained by ASAIO, ESLO and "SpecialtyCare" may provide insight about the relevant exposure, demographics, comorbidities, and clinical and laboratory variables that may predict outcome, aid selection of patients or even suggest futility (against the evidence presented here).

Research in context

\section{Evidence before this study}

ECMO appeared to benefit during the influenza A (H1N1) pandemic in 2009 and the middle east respiratory syndrome (MERS) starting in 2012. Patients with refractory ARDS showed that ECMO should be used as 
a rescue therapy because it is associated with lower mortality when compared to conventional mechanical ventilation. The role of ECMO in the management of ARDS related to the current COVID-19 pandemic remains debatable. The initial reports of using venovenous ECMO with COVID- 19 patients suffering from intractable hypoxemia observed a high mortality rate and recommended using ECMO with caution in the current pandemic. CENTRAL, MEDLINE/PubMed, Cochrane Library, and Scopus were systematically searched from inception to May 28, 2020, for terms related to COVID 19 and ECMO use with no language restrictions. The word search used was (COVID OR SARS COV2 OR pandemic) AND (ARDS) OR (acute respiratory distress syndrome) OR (acute lung injury) OR (respiratory failure) OR (respiratory insufficiency) OR (ECMO) OR (extracorporeal membrane oxygenation). Studies were included if they provided numerical or formal qualitative data on final ECMO outcomes in the current COVID 19 pandemic. None of the included studies involve prospective randomized analyses; and therefore, all the included studies were of low or moderate quality according to the Newcastle-Ottawa scale. However, because of the unique challenges and unprecedented timeline of this pandemic, it is not surprising that a prospective randomized analysis has not been performed assessing the role of ECMO in supporting patients with COVID-19. In fact, the investigators publishing the various studies reported in this systematic review are to be congratulated for their efforts to conduct this research during these challenging times.

\section{Added value of this systematic review and meta-analysis}

This systematic review is the first in the current pandemic to perform a meta-analysis from all available cohort studies using venovenous ECMO for acute respiratory distress system caused by severe COVID 19 infection. Across low-to-moderate quality studies, we noted a reduction in short term mortality with venovenous ECMO in comparison to what is known about treatment with conventional mechanical ventilation in severe COVID 19. Given the current challenges of doing large clinical trials in this population of COVID 19 critically ill patients, a large well designed randomised controlled trial of ECMO treatment for COVID 19 is unlikely to happen. Therefore, the results of this meta-analysis represent the most comprehensive and up-to-date synthesis of the available evidence for clinicians on the use of venovenous ECMO in COVID 19 patients with severe acute respiratory distress syndrome.

\section{Conclusions}

The study included the largest number of patients with outcome findings of ECMO in this current pandemic. Our findings showed that the use of venovenous ECMO at high-volume ECMO centres may be beneficial for selected COVID 19 patients with severe ARDS. However, none of the included studies involve prospective randomized analyses; and therefore, all the included studies were of low or moderate quality according to the Newcastle-Ottawa scale. In the current era and environment of the pandemic, it will likely be very challenging to conduct a prospective randomized trial of ECMO versus no-ECMO for COIVID-19. Therefore, the information contained in this systematic review of the literature is valuable and provides important guidance.

\section{Abbreviations}

ARDS : Adult respiratory distress syndrome, ECMO:Extracorporeal membrane oxygenation

ELSO : Extracorporeal life support organisation, MERS:Middle east respiratory syndrome

UFH : Unfractionated heparin, vvECMO : venovenous Extracorporeal membrane oxygenation

ASAIO : American society for artificial internal organs

Consent for publication: All authors consent for publication

\section{Author contribution}

HHE conceived the study, which was overseen by MG. ASH, TA, and HY devised the search strategy, executed the search, and selected the studies for inclusion. ASH, TH, HY and HHE extracted the data, 
which were analysed by MG. All authors were involved in development of the methodological approach. HHE, ASH, TA and HY wrote the Article, which was reviewed and revised by all authors.

Declaration of interests: NoneAcknowledgments: None

Availability of data and material: All data and material available on request

Ethics approval and consent to participate: Not applicable Funding: No funding source

Figures

Figure 1: Flow diagram of study selection

Figure 2: Forest plot of pooled analysis of mortality by random effect model in all studies of ECMO with COVID 19 patients

Figure 3: Forest plot of pooled analysis of mortality by random effect model in all studies of ECMO with COVID 19 patients with subgroup division of country of origin

Figure 4: Forest Plots for Odds of Mortality in COVID-19 Patients Receiving ECMO Therapy versus conventional invasive mechanical ventilation therapy.

Figure 5: Funnel plot of standard error

Tables

Table 1: Characteristics and quality of the included studies

Table 2: Pre ECMO patient characteristics

Table 3: Main outcome of ECMO patients

References

1 [The epidemiological characteristics of an outbreak of 2019 novel coronavirus diseases (COVID-19) in China]. Zhonghua Liu Xing Bing Xue Za Zhi 2020; 41 : 145-51.

2 WHO. Coronavirus disease (COVID-19) Situation Report - 107. World Heal Organ 2020; 2019 : 2633.

3 Surveillances V. The epidemiological characteristics of an outbreak of 2019 novel coronavirus diseases (COVID-19) in China. Zhonghua Liu Xing Bing Xue Za Zhi 2020; 41 : 145-51.

4. Zhou F, Yu T, Du R, et al. Clinical course and risk factors for mortality of adult inpatients with COVID-19 in Wuhan, China: a retrospective cohort study. Lancet 2020; 395: 1054-62.

5. Grasselli G, Zangrillo A, Zanella A, et al. Baseline Characteristics and Outcomes of 1591 Patients Infected With SARS-CoV-2 Admitted to ICUs of the Lombardy Region, Italy. JAMA 2020; published online April. DOI:10.1001/jama.2020.5394.

6. Richardson S, Hirsch JS, Narasimhan M, et al. Presenting Characteristics, Comorbidities, and Outcomes Among 5700 Patients Hospitalized With COVID-19 in the New York City Area. JAMA 2020; published online April. DOI:10.1001/jama.2020.6775.

7. Yan Y, Yang Y, Wang F, et al. Clinical characteristics and outcomes of patients with severe covid-19 with diabetes. BMJ open diabetes Res care 2020; 8. DOI:10.1136/bmjdrc-2020-001343.

8. Yu Y, Xu D, Fu S, et al. Patients with COVID-19 in 19 ICUs in Wuhan, China: a cross-sectional study. Crit Care 2020; 24: 219.

9. Chen T, Wu D, Chen H, et al. Clinical characteristics of 113 deceased patients with coronavirus disease 2019: retrospective study. BMJ 2020; 368. DOI:10.1136/bmj.m1091. 
10. The Australia and New Zealand Extracorporeal Membrane Oxygenation (ANZ ECMO) Influenza Investigators. Extracorporeal membrane oxygenation for 2009 influenza A(H1N1) acute respiratory distress syndrome. JAMA. 2009;(302)

11. Noah M.A., Peek G.J., Finney S.J. Referral to an Extracorporeal Membrane Oxygenation Center and Mortality Among Patients With Severe 2009 Influenza A(H1N1) JAMA. 2011;306:1659-1668.

12. Alshahrani M.S., Sindi A., Alshamsi F. Extracorporeal membrane oxygenation for severe Middle East respiratory syndrome coronavirus. Ann Intensive Care. 2018;8:3

13. Henry BM. COVID-19, ECMO, and lymphopenia: a word of caution. Lancet Respir Med 2020; 8: e24.

14. World Health Organization. WHO Clinical management of severe acute respiratory infection (SARI) when COVID-19 disease is suspected. Who 2020; 2019: 12 .

15. Stang A: Critical evaluation of the Newcastle-Ottawa scale for the assessment of the quality of nonrandomized studies in meta-analyses. Eur J Epidemiol 2010, 25: 603-605.

16. DerSimonian R, Laird N. Meta-analysis in clinical trials. Control Clin Trials 1986;7:177-88.

17. Higgins JP, Thompson SG, Deeks JJ, Altman DG. Measuring inconsistency in meta-analyses. BMJ. 2003;327: 557-560.

18. Zhou, Ning and Wang, Luyun and Jiang, Jiangang and Cheng, Peng and Cui, Guanglin and Wang, Feng and Guan, Zhimin and Zhang, Panpan and Li, Shengqing and Wen Wang, Dao, Application of ECMO Successfully Treated Critically Ill COVID-19 Patients (4/1/2020). Available at SSRN: https://ssrn.com/abstract $=3569889$

19. Zhang G, Hu C, Luo L, et al. Clinical Features and Treatment of 221 Patients with COVID-19 in Wuhan, China. SSRN Electron J 2020. DOI:10.2139/ssrn.3546095.

20. Takeda S. Nationwide system to centralize decisions around ECMO use for severe COVID-19 pneumonia in Japan. Acute Med Surg 2020; 4: 20-1.

21. Jacobs JP, Stammers AH, St Louis J, et al. Extracorporeal Membrane Oxygenation in the Treatment of Severe Pulmonary and Cardiac Compromise in COVID-19: Experience with 32 patients. ASAIO J 2020. DOI:10.1097/MAT.0000000000001185.

22. Yang $\mathrm{X}, \mathrm{Yu} \mathrm{Y}, \mathrm{Xu}$ J, et al. Clinical course and outcomes of critically ill patients with SARS-CoV-2 pneumonia in Wuhan, China: a single-centered, retrospective, observational study. Lancet Respir Med 2020; 2600: $1-7$.

23. Ruan Q, Yang K, Wang W, Jiang L, Song J. Correction to: Clinical predictors of mortality due to COVID-19 based on an analysis of data of 150 patients from Wuhan, China (Intensive Care Medicine, (2020), 10.1007/s00134-020-05991-x). Intensive Care Med 2020. DOI:10.1007/s00134-020-06028-z.

24. Li X, Guo Z, Li B, et al. Extracorporeal Membrane Oxygenation for Coronavirus Disease 2019 in Shanghai, China. ASAIO J 2020; : 1.

25. Guan W, Ni Z, Hu Y, et al. Clinical characteristics of coronavirus disease 2019 in China. N Engl J Med 2020; 382: 1708-20.

26. Zeng Y, Cai Z, Xianyu Y, Yang BX, Song T, Yan Q. Prognosis when using extracorporeal membrane oxygenation (ECMO) for critically ill COVID-19 patients in China: a retrospective case series. Crit Care 2020; 24: 148 .

27. Beyls C, Huette P, Abou-Arab O, Berna P, Mahjoub Y. Extracorporeal membrane oxygenation for COVID-19-associated severe acute respiratory distress syndrome and risk of thrombosis. Br J Anaesth 2020; : $1-3$. 
28. Osho AA, Moonsamy P, Hibbert KA, et al. Veno-venous Extracorporeal Membrane Oxygenation for Respiratory Failure in COVID-19 Patients: Early Experience From a Major Academic Medical Center in North America. Ann Surg 2020; : 440-53.

29. Haye G, Fourdrain A, Abou-Arab O, Berna P, Mahjoub Y. COVID-19 outbreak in France: Setup and activities of a mobile Extra Corporeal Membrane Oxygenation (ECMO) team during the first 3 weeks. J Cardiothorac Vasc Anesth 2020; 000: 8-10.

30. Namendys-Silva SA. ECMO for ARDS due to COVID-19. Heart Lung. 2020 Mar 26:S01479563(20)30100-X. doi: 10.1016/j.hrtlng.2020.03.012. Epub ahead of print.

31. Henry BM, Lippi G. Poor survival with extracorporeal membrane oxygenation in acute respiratory distress syndrome (ARDS) due to coronavirus disease 2019 (COVID-19): Pooled analysis of early reports. J Crit Care. 2020 Apr 1;58:27-28.

32. Barbaro RP, Odetola FO, Kidwell KM, Paden ML, Bartlett RH, Davis MM, Annich GM. Association of hospital-level volume of extracorporeal membrane oxygenation cases and mortality: analysis of the Extracorporeal Life Support Organization Registry. Am J Respir Crit Care Med 2015;191:894-901.

33. Force ARDSDT, Ranieri VM, Rubenfeld GD, Thompson BT, Ferguson ND, Caldwell E, Fan E, Camporota L, Slutsky AS. Acute respiratory distress syndrome: the Berlin definition. JAMA. 2012.

34. Gattinoni, L., Chiumello, D. \& Rossi, S. COVID-19 pneumonia: ARDS or not? 2020. Crit Care 24, 154

35. Yin S, Huang M, Li D, Tang N. Difference of coagulation features between severe pneumonia induced by SARS-CoV2 and non-SARS-CoV2. J Thromb Thrombolysis. 2020. ahead of print

36. Fina D, Matteucci M, Jiritano F, Meani P, Lo Coco V, Kowalewski M, Maessen J, Guazzi M, Ballotta A, Ranucci M, Lorusso R. Extracorporeal membrane oxygenation without therapeutic anticoagulation in adults: a systematic review of the current literature. Int J Artif Organs. 2020:391398820904372. ahead of print.

37. ELSO COVID-19 interim guidelines. ELSO, 2020 (Available from www.elso.org)

38. Munshi L, Walkey A, Goligher E, Pham T, Uleryk EM, Fan E. Venovenous extracorporeal membrane oxygenation for acute respiratory distress syndrome: a systematic review and meta-analysis. Lancet Respir Med. 2019;7(2):163-172.

39. Mehta P, McAuley DF, Brown M, Sanchez E, Tattersall RS, Manson JJ, HLH Across Specialty Collaboration, UK. COVID-19: consider cytokine storm syndromes and immunosuppression. Lancet. 2020 Mar 28; 395(10229):1033-1034.

40. Abrams D, Ferguson ND, Brochard L, Fan E, Mercat A, Combes A, Pellegrino V, Schmidt M, Slutsky AS, Brodie D. ECMO for ARDS: from salvage to standard of care? Lancet Respir Med. 2019 Feb; 7(2):108-110.

\begin{tabular}{|c|c|c|c|c|c|c|}
\hline Study (author and year) & Country & Design & Setting & NOS & Follow-up & Primary enc \\
\hline Zhou et al. (2020) (18) & China & Observational & Single center & 4 & In-hospital & Death \\
\hline Zhang et al. (2020) (19) & China & Observational & Single center & 5 & In hospital & Death \\
\hline Takeda et al. (2020) (20) & Japan & Observational & Multicenter & 6 & In-hospital & Death \\
\hline Jacobs et al. (2020) (21) & USA & Observational & Multicenter & 6 & In-hospital & Death \\
\hline Yang et al. (2020) (22) & China & Observational & Single center & 7 & In-hospital & Death \\
\hline Ruan et al. (2020) (23) & China & Observational & Single center & 7 & In hospital & Death \\
\hline $\mathrm{Li}$ et al. $(2020)(24)$ & China & Observational & Single center & 7 & In-hospital & Death \\
\hline Guan et al. (2020) (25) & China & Observational & Single center & 7 & In-hospital & Death \\
\hline Zeng et al (2020) (26) & China & Observational & Single center & 6 & In-hospital & Death \\
\hline Beyls et al (2020) (27) & France & Observational & Single center & 6 & 4 weeks after discharge & Death \\
\hline
\end{tabular}




\begin{tabular}{lllllll}
\hline Study (author and year) & Country & Design & Setting & NOS & Follow-up & Primary end \\
\hline Osho et al (2020) (28) & USA & Observational & Single centre & 6 & In hospital & Death \\
Haye et al (2020) $(29)$ & France & Observational & Single centre & 6 & In hospital & Death \\
\hline
\end{tabular}

Table 1: Characteristics and quality of the included studies; NOS = Newcastle Ottawa scale

\begin{tabular}{|c|c|c|c|c|c|}
\hline $\begin{array}{l}\text { Study (author } \\
\text { and year) }\end{array}$ & $\begin{array}{l}\text { Patients } \\
\text { admitted to } \\
\text { ICU }\end{array}$ & $\begin{array}{l}\text { Patients } \\
\text { receiving } \\
\text { ECMO (total } \\
=194 \text { ) }\end{array}$ & Age & $\begin{array}{l}\text { ARDS Berlin } \\
\text { grading }\end{array}$ & $\begin{array}{l}\text { Days of } \\
\text { ventilation pre } \\
\text { ECMO }\end{array}$ \\
\hline $\begin{array}{l}\text { Zhou et } \\
\text { al. }(2020)\end{array}$ & 59 & 5 & 55.6 & $\mathrm{~N} / \mathrm{A}$ & $5(1-20)$ \\
\hline $\begin{array}{l}\text { Zhang et } \\
\text { al. }(2020)\end{array}$ & 48 & 10 & 55 & GRADE II & $\mathrm{N} / \mathrm{A}$ \\
\hline $\begin{array}{l}\text { Takeda et } \\
\text { al. }(2020)\end{array}$ & $\mathrm{N} / \mathrm{A}$ & 26 & 71 & GRADE III & $3(0-9)$ \\
\hline $\begin{array}{l}\text { Jacobs et } \\
\text { al. }(2020)\end{array}$ & $\mathrm{N} / \mathrm{A}$ & 85 & 52 & GRADE III & $4(2.5-6.5)$ \\
\hline $\begin{array}{l}\text { Yang et } \\
\text { al. }(2020)\end{array}$ & $\mathrm{N} / \mathrm{A}$ & 6 & 59.7 & $\mathrm{~N} / \mathrm{A}$ & $\mathrm{N} / \mathrm{A}$ \\
\hline $\begin{array}{l}\text { Ruan et } \\
\text { al. }(2020)\end{array}$ & 62 & 7 & 50 & $\mathrm{~N} / \mathrm{A}$ & $\mathrm{N} / \mathrm{A}$ \\
\hline $\mathrm{Li}$ et al. (2020) & 20 & 8 & 64.25 & GRADE III & $6(0-21)$ \\
\hline $\begin{array}{l}\text { Guan et } \\
\text { al. }(2020)\end{array}$ & 37 & 5 & 47 & $\mathrm{~N} / \mathrm{A}$ & $\mathrm{N} / \mathrm{A}$ \\
\hline $\begin{array}{l}\text { Zeng et al } \\
(2020)\end{array}$ & 12 & 12 & 50.9 & $\mathrm{~N} / \mathrm{A}$ & $\mathrm{N} / \mathrm{A}$ \\
\hline $\begin{array}{l}\text { Beyls et al } \\
(2020)\end{array}$ & $\mathrm{N} / \mathrm{A}$ & 16 & 62 & $\mathrm{~N} / \mathrm{A}$ & $4(1.5-7.5)$ \\
\hline $\begin{array}{l}\text { Osho et al } \\
(2020)\end{array}$ & $\mathrm{N} / \mathrm{A}$ & 6 & 47 & $\mathrm{~N} / \mathrm{A}$ & $5.5(3.5-6.75)$ \\
\hline $\begin{array}{l}\text { Haye et al } \\
(2020)\end{array}$ & $\mathrm{N} / \mathrm{A}$ & 8 & 57.2 & GRADE III & $4(1-9)$ \\
\hline
\end{tabular}

Table 2: Pre ECMO patient characteristics N/A: Not available

Table 3: Main outcome of ECMO patients

\begin{tabular}{|c|c|c|c|c|}
\hline $\begin{array}{l}\text { Study (author } \\
\text { and year) }\end{array}$ & $\begin{array}{l}\text { Veno venous } \\
\text { ECMO }\end{array}$ & $\begin{array}{l}\text { Duration on } \\
\text { ECMO (days) }\end{array}$ & $\begin{array}{l}\text { Patient endpoint } \\
\text { (weaned/death) } \\
\text { (total=136) }\end{array}$ & $\begin{array}{l}\text { Mortality from } \\
\text { ECMO }\end{array}$ \\
\hline Zhou et al. (2020) & $100 \%$ & $10(5-16)$ & 5 & $0(0 \%)$ \\
\hline $\begin{array}{l}\text { Zhang et } \\
\text { al. }(2020)\end{array}$ & $100 \%$ & $\mathrm{~N} / \mathrm{A}$ & 5 & $3(60 \%)$ \\
\hline $\begin{array}{l}\text { Takeda et } \\
\text { al. }(2020)\end{array}$ & $100 \%$ & $\mathrm{~N} / \mathrm{A}$ & 16 & $0(0 \%)$ \\
\hline $\begin{array}{l}\text { Jacobs et } \\
\text { al. }(2020)\end{array}$ & $100 \%$ & $\mathrm{~N} / \mathrm{A}$ & 50 & $21(42 \%)$ \\
\hline Yang et al. (2020) & $100 \%$ & $\mathrm{~N} / \mathrm{A}$ & 5 & $5(100 \%)$ \\
\hline
\end{tabular}




\begin{tabular}{lllll}
\hline $\begin{array}{l}\text { Study (author } \\
\text { and year) }\end{array}$ & $\begin{array}{l}\text { Veno venous } \\
\text { ECMO }\end{array}$ & $\begin{array}{l}\text { Duration on } \\
\text { ECMO (days) }\end{array}$ & $\begin{array}{l}\text { Patient endpoint } \\
\text { (weaned/death) } \\
\text { (total=136) }\end{array}$ & $\begin{array}{l}\text { Mortality from } \\
\text { ECMO }\end{array}$ \\
\hline Ruan et al. $(2020)$ & $100 \%$ & N/A & 7 & $7(100 \%)$ \\
Li et al. $(2020)$ & $100 \%$ & $37(9-47)$ & 6 & $3(50 \%)$ \\
Guan et al. $(2020)$ & $100 \%$ & N/A & 5 & $5(100 \%)$ \\
Zeng et al (2020) & $100 \%$ & $11(3-28)$ & 8 & $5(63 \%)$ \\
Beyls et al $(2020)$ & $100 \%$ & $16(2-28)$ & 16 & $4(25 \%)$ \\
Osho et al $(2020)$ & $100 \%$ & $12(4-18)$ & 6 & $2(33 \%)$ \\
Haye et al (2020) & $100 \%$ & $14(8-28)$ & 7 & $2(29 \%)$ \\
\hline
\end{tabular}

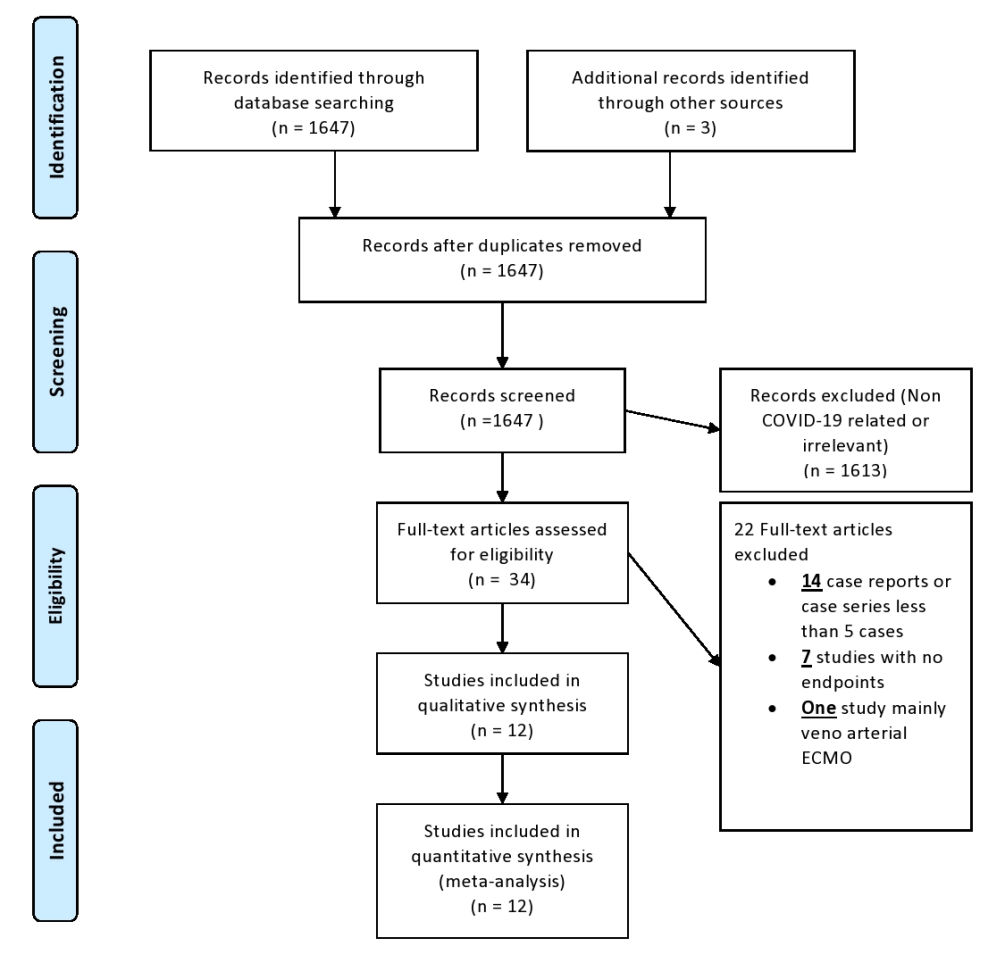

Figure 1: Flow diagram of study selection (PRISMA) 

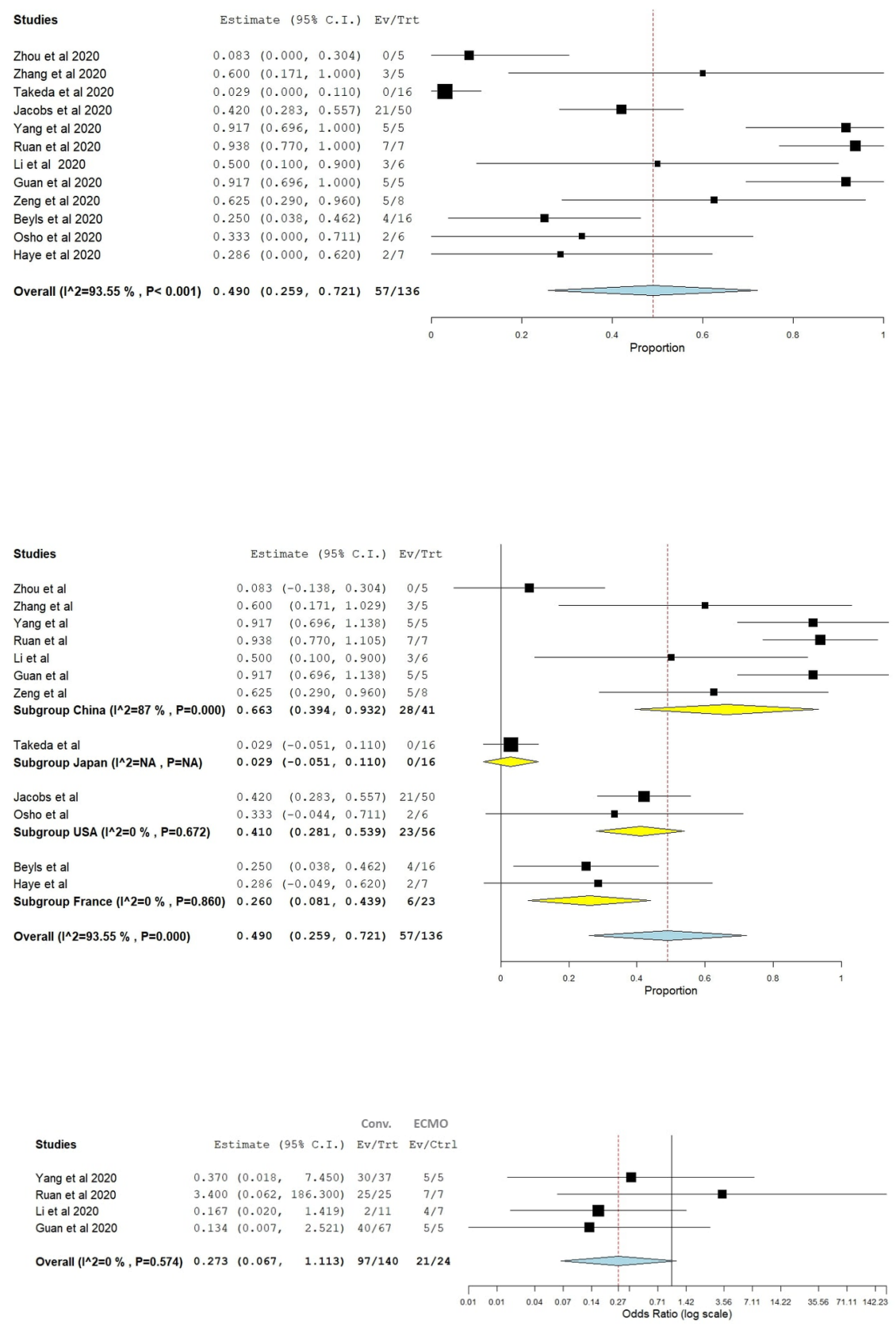


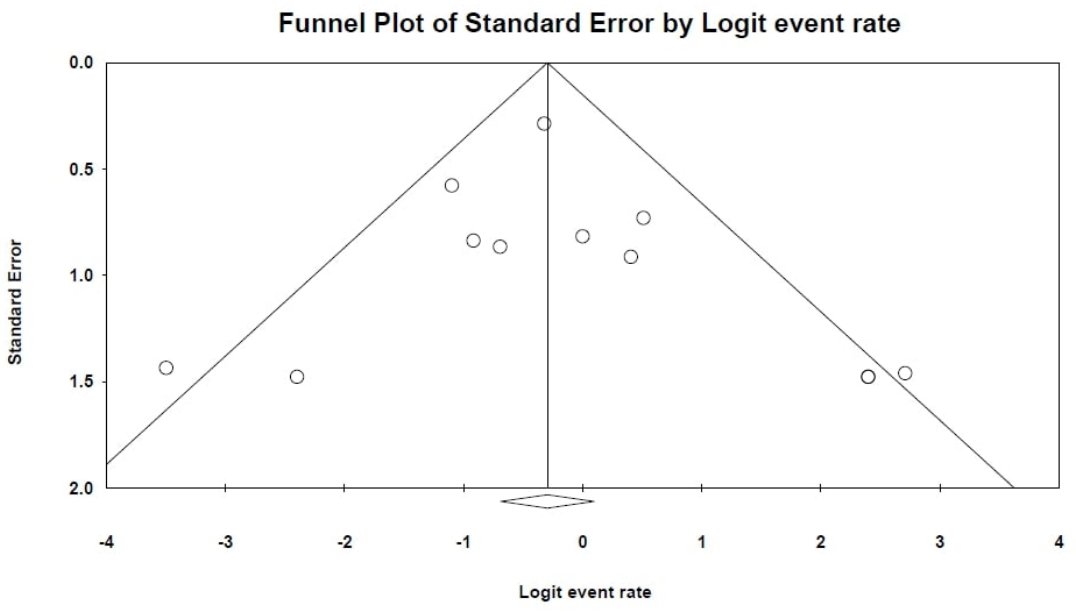

